\title{
Natural selection and genetic diversity of domain I of Plasmodium falciparum apical membrane antigen-1 on Bioko Island
}

\author{
Ya-Nan Wang ${ }^{1 \dagger}$, Min Lin ${ }^{2 \dagger}$, Xue-Yan Liang ${ }^{1,2}$, Jiang-Tao Chen ${ }^{3,4}$, Dong-De Xie ${ }^{3}$, Yu-Ling Wang ${ }^{3}$, \\ Carlos Salas Ehapo ${ }^{5}$, Urbano Monsuy Eyi ${ }^{5}$, Hui-Ying Huang ${ }^{1,2}$, Jing-Li Wu ${ }^{6}$, Dan-Yan Xu' ${ }^{6}$, Zhi-Mao Chen ${ }^{6}$, \\ Yi-Long $\mathrm{CaO}^{6}$ and Hai-Bin Chen ${ }^{1 *}$
}

\begin{abstract}
Background: Plasmodium falciparum apical membrane antigen-1 (PfAMA-1) is a promising candidate antigen for a blood-stage malaria vaccine. However, antigenic variation and diversity of PfAMA-1 are still major problems to design a universal malaria vaccine based on this antigen, especially against domain I (DI). Detail understanding of the PfAMA-1 gene polymorphism can provide useful information on this potential vaccine component. Here, general characteristics of genetic structure and the effect of natural selection of Dls among Bioko P. falciparum isolates were analysed.

Methods: 214 blood samples were collected from Bioko Island patients with P. falciparum malaria between 2011 and 2017. A fragment spanning DI of PfAMA-1 was amplified by nested polymerase chain reaction and sequenced. Polymorphic characteristics and the effect of natural selection were analysed using MEGA 5.0, DnaSP 6.0 and Popart programs. Genetic diversity in 576 global PfAMA-1 Dls were also analysed. Protein function prediction of new amino acid mutation sites was performed using PolyPhen-2 program.

Results: 131 different haplotypes of PfAMA-1 were identified in 214 Bioko Island P. falciparum isolates. Most amino acid changes identified on Bioko Island were found in C1L. 32 amino acid changes identified in PfAMA-1 sequences from Bioko Island were found in predicted RBC-binding sites, B cell epitopes or IUR regions. Overall patterns of amino acid changes of Bioko PfAMA-1 Dls were similar to those in global PfAMA-1 isolates. Differential amino acid substitution frequencies were observed for samples from different geographical regions. Eight new amino acid changes of Bioko island isolates were also identified and their three-dimensional protein structural consequences were predicted. Evidence for natural selection and recombination event were observed in global isolates.

Conclusions: Patterns of nucleotide diversity and amino acid polymorphisms of Bioko Island isolates were similar to those of global PfAMA-1 Dls. Balancing natural selection across Dls might play a major role in generating genetic diversity in global isolates. Most amino acid changes in Dls occurred in predicted B-cell epitopes. Novel sites mapped on a three dimensional structure of PfAMA-1 showed that these regions were located at the corner. These results may provide significant value in the design of a malaria vaccine based on this antigen.
\end{abstract}

Keywords: Bioko Island, Plasmodium falciparum, AMA-1, Domain I, Natural selection, Genetic diversity

\footnotetext{
*Correspondence: chenhb@stu.edu.cn

†Ya-Nan Wang and Min Lin contributed equally to this work

${ }^{1}$ Department of Histology and Embryology, Shantou University Medical

College, Shantou, Guangdong, People's Republic of China

Full list of author information is available at the end of the article
} 


\section{Background}

Although global morbidity and mortality have decreased substantially, malaria is still a great public health concern, especially in Africa [1]. The World Malaria Report 2018 draws on data from 87 countries and areas with ongoing malaria transmission. Recent research shows that after an unprecedented period of success in global malaria control, progress has stalled. An estimated 219 million (95\% confidence interval [CI]: 203-262 million) persons suffer from malaria infections worldwide, with 435,000 malaria deaths in 2017. Fifteen countries accounted for $80 \%$ of global malaria deaths in 2017 , and the 10 highest burdened African countries saw an estimated 3.5 million more malaria cases in 2017 compared with the previous year [2]. Malaria is endemic in Equatorial Guinea, a country in Central West Africa with a population of around 1 million inhabitants. In 2004, the government and private parties formed a Public-Private Partnership that has spent the last 15 years controlling malaria on Bioko Island and parts of the mainland in an effort to reduce malaria's burden on the population. The Bioko Island Malaria Control Project (BIMCP) implemented by the U.S. NGO, Medical Care Development International (MCDI) and the Ministry of Health and Social Welfare of the Government of Equatorial Guinea, has reduced malaria prevalence from $74 \%$ (by thick blood smear) in 2003 to $11 \%$ (by rapid diagnostic test) in 2017 in children 2 to 14 years of age. Infant mortality due to malaria infection has reduced by $85 \%$ (by rapid diagnostic test). However, it has proven difficult to eliminate malaria from this region despite the increasing intensity of malaria intervention. With the emergence and geographical expansion of anti-malarial resistance worldwide, molecular markers are essential tools for the surveillance of resistant Plasmodium parasites [3]. The decrease of island-wide $P$. falciparum prevalence was steep in the first year following the implementation of malaria intervention. However, it is still a major obstacle to public health and economic growth for countries in the tropics and subtropical regions [4].

Vaccines are the most cost effective and efficient method of protecting against malaria. However, malaria, one of the oldest and deadliest pathogens in human history, remains without a marketed vaccine. The EquatoGuinean Malaria Vaccine Initiative (EGMVI) is engaging in conducting a series of clinical trials that will advance a PfSPZ vaccine through to phase III clinical trials and eventually test the public health utility of the vaccine in malaria elimination projects. Therefore, the development of an effective vaccine against $P$. falciparum is a necessary priority, particularly due to the increased resistance of this parasite to anti-malarial drugs $[3,5]$. Up to date, several candidate proteins, including circumsporozoite protein (CSP), Duffy-binding protein (DBP), merozoite surface protein-1 (MSP-1), apical membrane antigen-1 (AMA-1), and thrombospondin-related anonymous protein (TRAP) have been tested for their potential as candidate antigens for the development of effective vaccines [6]. For MSP-1, previous report showed high genetic diversity and MOI values among the $P$. falciparum population [7]. A high prevalence of Pfdhfr-Pfdhps quadruple mutations were detected, which is associated with sulfadoxine resistance in P. falciparum isolates on Bioko Island. This result reflects both the high endemic level of malaria and its transmission on Bioko Island [8].

AMA-1 is an 83-kDa type I integral membrane protein localized to the apical complex [9]. It is mainly expressed in the merozoite and sporozoite stages of malaria parasites and appears to be transported to the merozoite surface in late schizonts and free merozoites [9-14]. AMA-1 consists of a signal sequence, a cysteine-rich ectodomain, a conserved cytoplasmic region and a transmembrane region [15]. The ectodomain of AMA-1 is subdivided into three immunogenic domains, and natural immune responses against this domain have been reported in patients infected with $P$. falciparum $[16,17]$. The biological function of AMA-1 is not understood thoroughly, though its stage-specific expression and localization suggest that this protein might play a crucial role in invasion $[9,12,18,19]$. There is also several evidence that suggest that AMA-1 forms a complex with RON2 to form the moving junction during invasion of merozoites into erythrocytes [17-23]. According to a previous report, AMA-1 can induce a protective immune response that produces antibodies that can effectively inhibit $P$. falciparum from invading red blood cells [24]. However, polymorphism of protozoan antigen and immune evasion of plasmodium have impeded the development of a malaria vaccine. PfAMA1 is the main candidate antigen of a red-blood stage vaccine and has entered a phase II clinical trial [25]. Due to its essential role for parasite survival and high level of immunogenicity during natural infection in humans, vaccination studies conducted in BALB/C mouse models have confirmed that AMA-1 is a potential vaccine antigen against $P$. falciparum [26].

Independent studies provide strong evidence that balancing selection acts to maintain these polymorphisms in the population, reflecting the importance of AMA-1 as a target of protective immunity [27-30]. However, these antibodies can recognize either conserved or allelespecific epitopes of AMA-1, resulting in limited protection for different alleles [31]. Several polymorphisms can be observed in DI of AMA-1, suggesting that this region seems to be the main target of anti-AMA-1 protective antibodies [30, 32-35]. Nevertheless, genetic diversity of AMA-1 among Plasmodium field isolates and the 
presence of variant forms in different geographic areas present hurdles in successful malaria vaccine design. Therefore, in order to design an efficient and protective malaria vaccine, it is essential to monitor genetic variations of candidate vaccine antigens in global malaria isolates circulating in endemic areas [36].

Although the incidence of malaria has decreased significantly through BIMCP, P. falciparum is still the most critical concern for malaria control and prevention on Bioko Island with the emergence of artemisinin resistance $[3,5]$. In this study, natural selection and genetic diversity of AMA-1 DI region in Bioko P. falciparum isolates were analysed.

\section{Methods}

\section{Study area}

The study was carried out in the clinic of the Chinese medical aid team for the Republic of Equatorial Guinea, Malabo Regional Hospital. Ethical approval was obtained from the Ethics Committee of Malabo Regional Hospital (EGCNGD-071). Bioko Island, the largest island of Equatorial Guinea, is located in the Gulf of Guinea, approximately $100 \mathrm{~km}$ off the coast of southern Nigeria and $160 \mathrm{~km}$ northwest of continental Equatorial Guinea
(Fig. 1). The island has a population of 334,463 inhabitants (2015 census, of which approximately $90 \%$ live in Malabo, the capital city of Equatorial Guinea) and a humid tropical environment.

\section{Sample collection}

Plasmodium falciparum clinical samples (from individuals 4 months to 80 years of age) were collected from 214 confirmed $P$. falciparum malaria cases identified by microscopic examination and an immuno-colloidal gold test kit (ICT Diagnostics) in 2011-2017. Informed consent was obtained from all participating subjects or their parents. Blood samples were collected on filter paper (Whatman $3 \mathrm{~mm}$, GE Healthcare, Pittsburg, USA) for further molecular analysis, air-dried and stored in sealed plastic bags at ambient temperature. Thick blood smears were air dried and stained with $10 \%$ fresh Giemsa following standard procedures. After coding and recording the patient medical records, the dried blood filters were then stored in plastic sealing bags and stored at $-80^{\circ} \mathrm{C}$.

\section{DNA extraction}

Parasite DNA was extracted from dried filter blood spots by following the Chelex-100 extraction method described

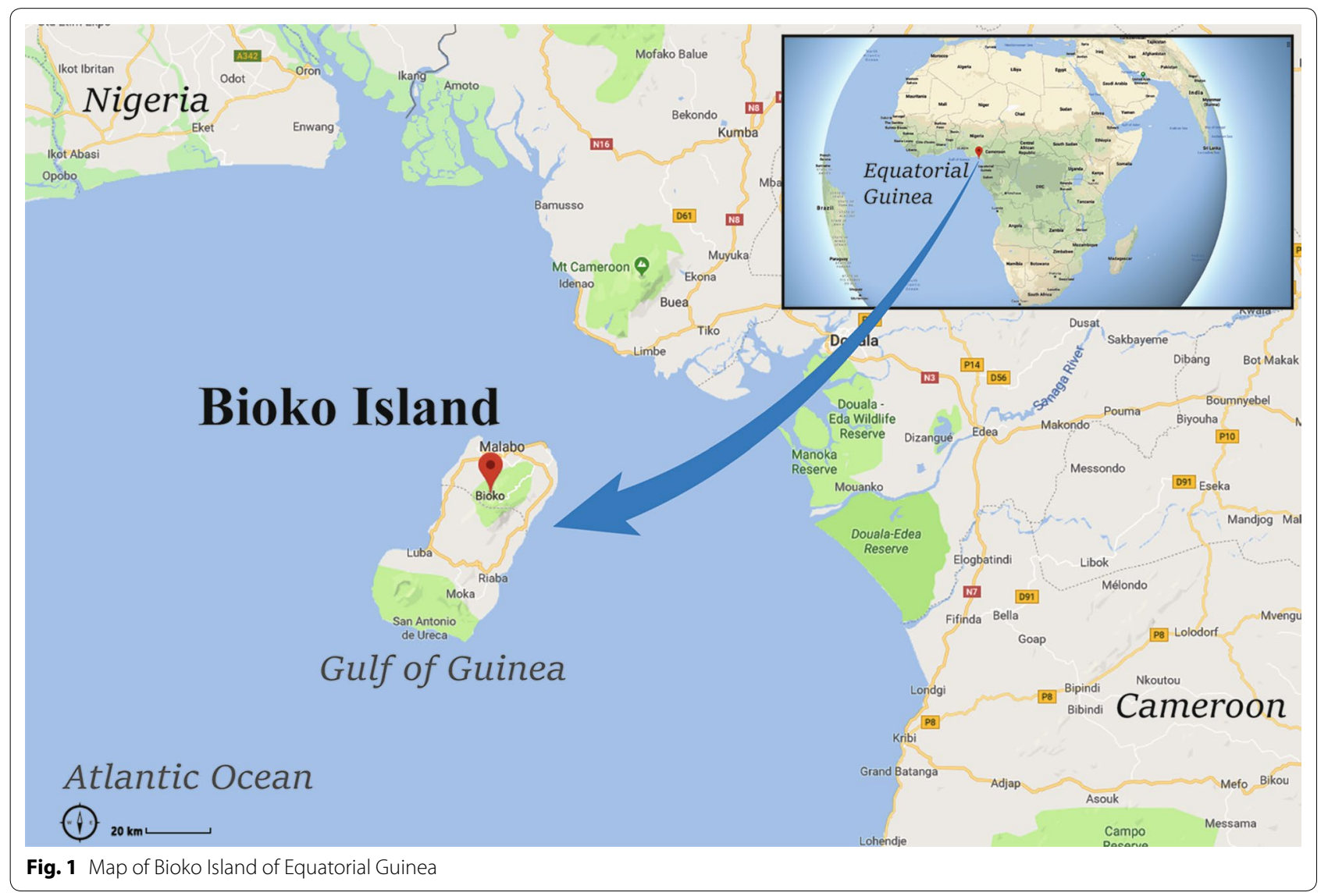


in previous article [3]. The DNA products were collected in sterile tubes and stored at $-20^{\circ} \mathrm{C}$.

\section{Amplification and sequencing analysis of DI of PfAMA-1}

AMA-1 sequences were amplified by nested PCR. For the first round PCR, $1 \mu \mathrm{l}$ of DNA was added to $12.5 \mu \mathrm{l}$ $2 \times$ MasterMix (DNA polymerase, dNTP mixture, PCR buffer), $1 \mu \mathrm{l}$ of $10 \mu \mathrm{mol} / \mathrm{l}$ forward primer (5'-TGAAGA AGTTCATGGTTCAGGT-3'), $1 \mu \mathrm{l}$ of $10 \mu \mathrm{mol} / \mathrm{l}$ reverse primer (5'-GCACTTTTGATCATACTAGCGTT-3'), and sterile ultrapure water to a final volume of $25 \mu$ l. Thermal cycling parameters for PCR were as follows: one cycle of an initial denaturation at $94{ }^{\circ} \mathrm{C}$ for $3 \mathrm{~min}, 30$ cycles of $94{ }^{\circ} \mathrm{C}$ for $30 \mathrm{~s}$, annealing at $55^{\circ} \mathrm{C}$ for $30 \mathrm{~s}$ and extension at $72{ }^{\circ} \mathrm{C}$ for $2 \mathrm{~min}$, followed by a final extension step at $72^{\circ} \mathrm{C}$ for $5 \mathrm{~min}$. For the second round PCR, $2 \mu \mathrm{l}$ of the primary PCR product was amplified in a $50 \mu$ reaction volume comprised of $25 \mu \mathrm{l} 2 \times$ Master Mix (DNA polymerase, dNTP mixture, PCR buffer), $2 \mu \mathrm{l}$ of $10 \mu \mathrm{mol} / \mathrm{l}$ forward primer (5'-GTTGATCCGAAGCACTCA-3'), $2 \mu \mathrm{l}$ of 10 $\mu \mathrm{mol} / \mathrm{l}$ reverse primer $\left(5^{\prime}\right.$-AGATGCTGAAGTAGCTGG AA- $\left.3^{\prime}\right)$, and sterile ultrapure water to a final volume of $50 \mu \mathrm{l}$. All PCR products were analysed using $2.0 \%$ agarose gel electrophoresis, and then, they were purified and sequenced by using an ABI $3730 \times \mathrm{L}$ Automated Sequencer (Shanghai Yingjun Biotechnology Co., LTD, Guangzhou branch). In our study, DI (nt: $445 \sim 906$ bp; 149-302 aa) sequences of the PfAMA-1 gene in 214 $P$. falciparum isolates were successfully amplified, including the $\mathrm{C} 1$ region (559-693 bp; $187-207 \mathrm{aa}$ ), C1L region (586-621 bp; 197-207 aa), C2 region (724-735 bp; 242-245 aa and 844-858 bp; 282-286 aa) and C3 region (514-525 bp; 172-175 aa). To ensure the accuracy of the sequencing, at least two clones of each isolate was sequenced. Sequencing primers were the reverse primers of the second round PCR, all sequences were analysed and integrated by Bioedit and MEGA5.0 software. These nucleotide sequences have been deposited at SRA under accession numbers (SRX4999365-SRX4999578).

\section{Statistical analysis \\ Nucleotide sequence polymorphism analysis and neutrality test}

Nucleotide and predicted amino acid sequences of DI were analysed using MEGA5.0 [37]. Nucleotide sequence polymorphism analysis was conducted for 214 sequences [37]. Numbers of segregating sites (S), haplotypes $(\mathrm{H})$, haplotype diversity $(\mathrm{Hd})$, nucleotide diversity $(\pi)$, and average number of pair-wise nucleotide differences within a population $(\mathrm{K})$ were estimated using DnaSP6.0 [38]. The value of $\pi$ was calculated to estimate step-wise diversity throughout DI based on a sliding window of 100 bases with a step size of $5 \mathrm{bp}$.
Values of non-synonymous ( $\mathrm{dN})$ and synonymous (dS) substitutions were estimated and compared using the $\mathrm{Z}$ test $(P<0.05$ was considered significant $)$ in the MEGA5.0 program [37] based on method of Nei and Gojobori [39] with Jukes and Cantor correction. Tajima's D value [40] and Fu and Li's D and F values [41] were analysed using DnaSP 6.0 to evaluate the neutral theory of evolution [38]. Recombination parameters $(\mathrm{R})$, which included the effective population size and probability of recombination between adjacent nucleotides per generation, and minimum number of recombination events $(\mathrm{Rm})$ were analysed by DnaSP6.0 [38]. Linkage disequilibrium (LD) between different polymorphic sites was computed based on the $\mathrm{R}^{2}$ index using DnaSP6.0 [38]. Protein function prediction of new amino acid mutation sites was performed using the PolyPhen-2 program [42].

\section{Population diversity of PfAMA-1 Dls among other global $P$. falciparum isolates}

Genetic diversity of PfAMA-1 DIs in other global P. falciparum isolates was analysed. Parasite populations from Ghana ( $\mathrm{n}=37$, AB71569-AB715734), Tanzania $(\mathrm{n}=62$, AB715636-AB715697), Nigeria $(n=51, A J 408300-$

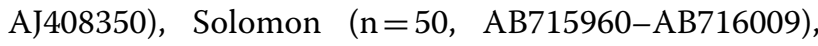
Gambia $(\mathrm{n}=114$, FJ555752-FJ555865), Kenya $(\mathrm{n}=129$, FN869569-FN869697), Benin $(\mathrm{n}=23, \quad$ AJ271168-

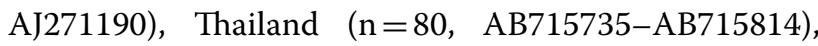
and Venezuela $(\mathrm{n}=30$, EU332414-EU332443) were included in the analysis. All publicly available sequences covered the DI sequences of PfAMA-1. Nucleotide sequence polymorphism analysis and neutrality test for each population were performed using programs DnaSP6.0 [38] and MEGA5.0 [37] as described above. Genetic differentiation among parasite populations was calculated based on the fixation index (Fst) to estimate pairwise DNA sequence diversity between and within populations using Arlequin 3.5 [43]. To investigate relationships among PfAMA-1 haplotypes, the haplotype network for a total of 790 PfAMA-1 sequences, including 214 Bioko Island sequences and the 576 publicly available sequences from Benin, Gambia, Ghana, Kenya, Nigeria, Solomon, Tanzania, Thailand and Venezuela, was constructed using the Median Joining algorithm of Popart program [44]. To assess whether genetic diversity in DI within P. falciparum isolates was associated with host immune pressure, genetic diversity in predicted B-cell epitopes, intrinsically unstructured/disordered regions (IUR), and RBC binding regions in other global PfAMA-1 were analysed [45-48]. Nucleotide diversity and natural selection of each region were analysed using DnasP6.0 as described above [38]. 


\section{Results}

Sequence polymorphism in DI sequences of PfAMA-1 on Bioko Island

One hundred thirty-one different haplotypes of DI were identified in 214 Bioko P. falciparum isolates. Comparison of 214 DI sequences with PfAMA-1 sequences from the 3D7 P. falciparum clone (GenBank Accession Number U65407) revealed amino acid changes at 40 positions in 214 Bioko PfAMA-1 sequences, including 26 di-morphic amino acid changes and 14 tri-, tetra-, or pentamorphic amino acid changes (Additional file 1). Most amino acid changes were in $\mathrm{C} 1$ region (14 amino acid changes), and these amino acid changes were distributed throughout each Bioko PfAMA-1 haplotype. Of the 14 tri-, tetra-, or penta-morphic amino acid changes, eight (E187K/N/D, L189P/H, D196N/K, E197Q/G/H/D/R/C, $\mathrm{H} 200 \mathrm{~L} / \mathrm{D} / \mathrm{R} / \mathrm{V} / \mathrm{G}, \mathrm{F} 201 \mathrm{~L} / \mathrm{S}, \mathrm{D} 204 \mathrm{~N} / \mathrm{K}$ and K230Q/E) were found in $\mathrm{C} 1$ region, whereas others $(\mathrm{C} 149 \mathrm{~K} / \mathrm{Y} / *$, G172E/V, Y175D/H, K243E/N, K245E/N, I282K/E) were found primarily in the $\mathrm{C} 2$ and $\mathrm{C} 3$ regions. Among 26 dimorphic amino acid changes identified in Bioko PfAMA-1 isolates, 18 have been previously reported in other geographical P. falciparum isolates. However, the remaining 8 changes P150T (2.29\%), V151I (0.76\%), I158S (1.53\%), G180C (2.29\%), A182V (0.76\%), D266N (3.82\%), S272N (0.76\%) and D281H (1.53\%) were novel (Additional file 1). Function prediction, using the PolyPhen-2 program, of the 8 novel sites showed that all 8 mutations were likely to affect the structure and function of PfAMA-1 (Table 5). The protein structural bioinformatics analysis inferred that these 8 mutations distributing the corner area of PfAMA-1 protein (Fig. 2).

\section{Amino acid polymorphisms in Dls of Bioko Island PfAMA-1 compared to global PfAMA-1 isolates}

When patterns of amino acid polymorphisms identified in DI of Bioko Island PfAMA-1 isolates were compared to those from other countries, similar but not identical polymorphic patterns were observed. Most amino acid changes of DI, previously identified in the global PfAMA-1 sequence databank, were found in the $\mathrm{C} 1$ region (Fig. 3). Most notable amino acid changes identified in Bioko PfAMA-1 DI were Y175D/H (92.37\%), E197H/R/D/G/Q/C (92.37\%), which present in Bioko Island sequences commonly, meanwhile their frequencies were also high in other global PfAMA-1 isolates. Frequencies of $\mathrm{Y} 175 \mathrm{D} / \mathrm{H}$ variation in other global isolates were as follows: Benin (80.95\%), Gambia (92.00\%), Ghana (95.24\%), Kenya (92.06\%), Nigeria (97.06\%), Solomon (87.50\%), Tanzania (91.18\%), Thailand (77.78\%), and Venezuela (40.00\%). Substitutions E197H/R/D/G/Q/C were also high in $\mathrm{C} 1 \mathrm{~L}$ region from most countries: Benin
(76.19\%), Gambia (88.00\%), Ghana (90.48\%), Kenya (93.65\%), Nigeria (94.12\%), Solomon (87.50\%), Tanzania $(85.30 \%)$, and Thailand $(94.45 \%)$, but not Venezuela (60.00\%). High frequencies of H200D/L/R/V/G (77.09\%), K206E (83.97\%), I225 N (77.86\%) and I282 K/E (90.83\%) variations were also observed in $\mathrm{C} 1$ and $\mathrm{C} 2$ regions of Bioko PfAMA-1 isolates. Highly polymorphic patterns of these four amino acid changes were also present in other global PfAMA-1 isolates. Two amino acid changes (D244 N, N286D) in C2 region were common in some of the African PfAMA-1 sequences (Gambia, Kenya, and Tanzania), but they were not identified or very rarely identified in the DI region from other countries. Moreover, P150T, V151I, I158S, G180C, A182 V, D266 N, S272 N, D281H mutations were uniquely identified in Bioko PfAMA-1 isolates, although their substitution frequencies were very low.

\section{Nucleotide diversity and natural selection of DIs from Bioko $P$. falciparum isolates}

Nucleotide diversity and natural selection of DIs from 214 Bioko P. falciparum isolates were analysed (Table 1). The $\mathrm{K}$ value was 12.795 . The highest nucleotide differences were found in $\mathrm{C} 1$ region $(\mathrm{K}=7.489)$, whereas the lowest were found in $\mathrm{C} 3$ region $(\mathrm{K}=0.925)$. Haplotype diversity for DIs was $0.991 \pm 0.0017$. This value was higher for the $\mathrm{C} 1$ region $(0.976 \pm 0.003)$ than for the $\mathrm{C} 2(0.599 \pm 0.031$ and $0.777 \pm 0.018)$ or $\mathrm{C} 3(0.692 \pm 0.020)$ region. The $\pi$ value of DIs was $0.02776 \pm 0.00042$. Analysis of $\pi$ values for the $\mathrm{C} 1, \mathrm{C} 1 \mathrm{~L}, \mathrm{C} 2$, and $\mathrm{C} 3$ regions revealed that the most nucleotide diversity was concentrated in the $\mathrm{C} 1 \mathrm{~L}$ and $\mathrm{C} 2$ regions. To examine whether natural selection has contributed to generation of DI diversity in Bioko $P$. falciparum populations, the value of $\mathrm{dN}-\mathrm{dS}$ was estimated using the Nei and Gojobori method. The value of $\mathrm{dN}-\mathrm{dS}$ for DIs was 0.0327 , suggesting that balancing natural selection might have occurred in DIs of the Bioko P. falciparum populations. Considering high positive $\mathrm{dN}-\mathrm{dS}$ values for the $\mathrm{C} 1 \mathrm{~L}(0.1378)$ and $\mathrm{C} 2(0.1669)$ regions, these regions might experience the most pressure from balancing natural selection forces. The estimated Tajima's D value of DIs was $0.56734(P>0.10)$. When Tajima's D value was analysed for each domain, the C1 (1.49861, $P>0.10)$ and C1L $(1.24277, P>0.10)$ regions showed higher positive Tajima's D values compared to other regions.

\section{Nucleotide diversity and natural selection of DIs in other global $P$. falciparum isolates}

Nucleotide diversity of DIs among other global isolates, including Benin, Gambia, Ghana, Kenya, Nigeria, Solomon, Tanzania, Thailand and Venezuela were analysed, and compared to Bioko PfAMA-1 isolates. $\mathrm{K}$ 

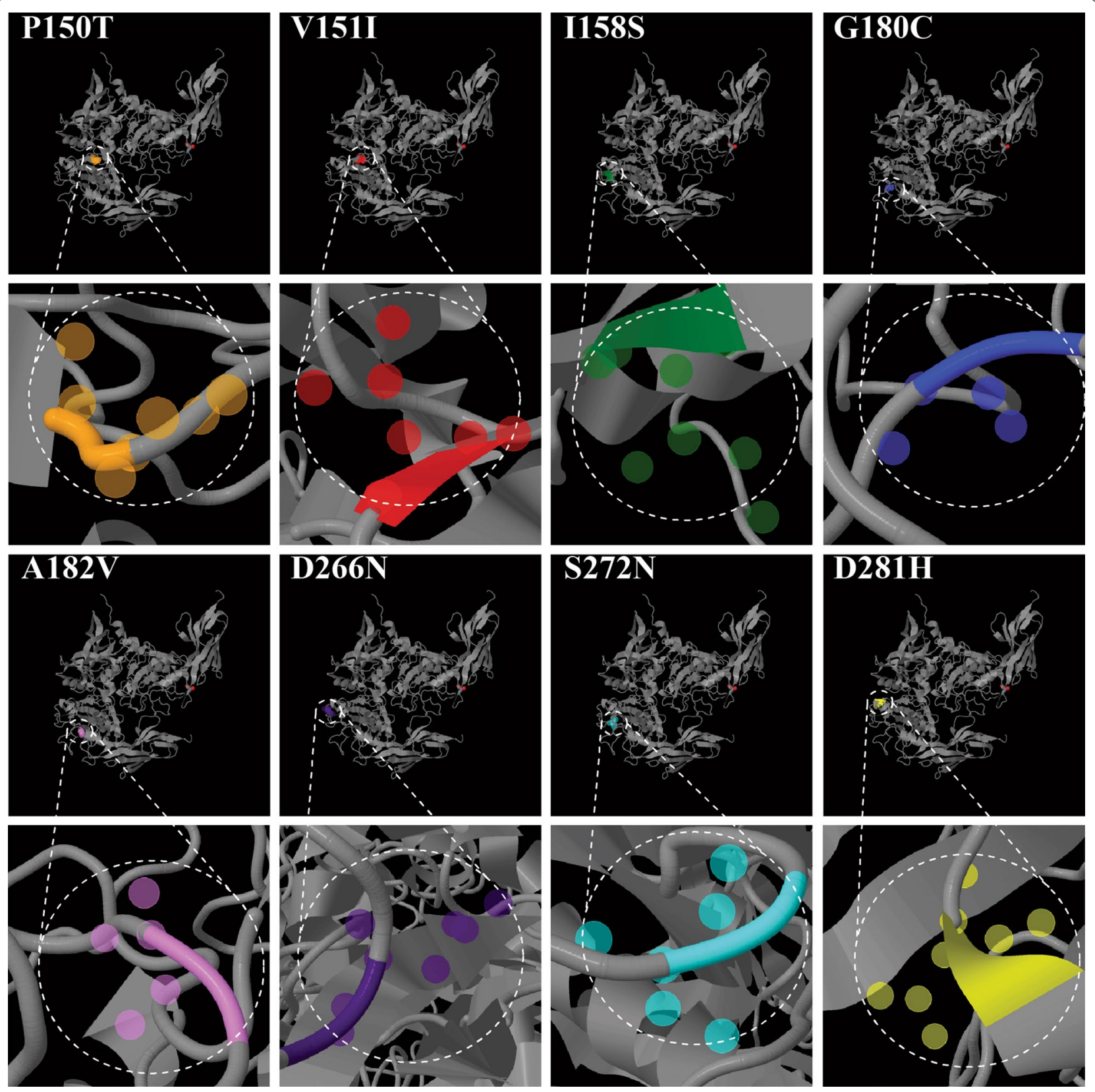

Fig. 2 Predicted three-dimensional structure of 8 novel sites

values of DIs among African PfAMA-1 isolates (Ghana, $K=12.555$; Kenya, $K=12.886)$ were higher than those of Asian (Thailand, $K=11.428$ ) and South American (Venezuela, $\mathrm{K}=5.617$ ) PfAMA-1 isolates (Table 2). Nucleotide diversity across DIs from different countries also slightly differed by geographical area. The level of nucleotide diversity across the DIs of Bioko Island P. falciparum isolates $(\pi=0.02776)$ was higher than that from Venezuela $(\pi=0.01216)$ or Thailand $(\pi=0.02474)$ isolates, but similar to that from Ghana $(\pi=0.02717)$, Benin $(\pi=0.02577)$,
Tanzania $(\pi=0.02697)$ and Solomon $(\pi=0.02552)$. A sliding window plot of $\pi$ revealed that DIs from different geographical areas shared highly similar patterns of nucleotide diversity through their sequences, with two peaks in the $\mathrm{C} 1$ region. Maximum diversity was found in the $\mathrm{C} 1$ region (Fig. 4a). All DI sequences from different countries showed positive Tajima's D values, suggesting a pattern of balancing selection across DIs in global P. falciparum samples (Table 2). A sliding window plot analysis also showed that DIs of other global PfAMA-1 genes had 


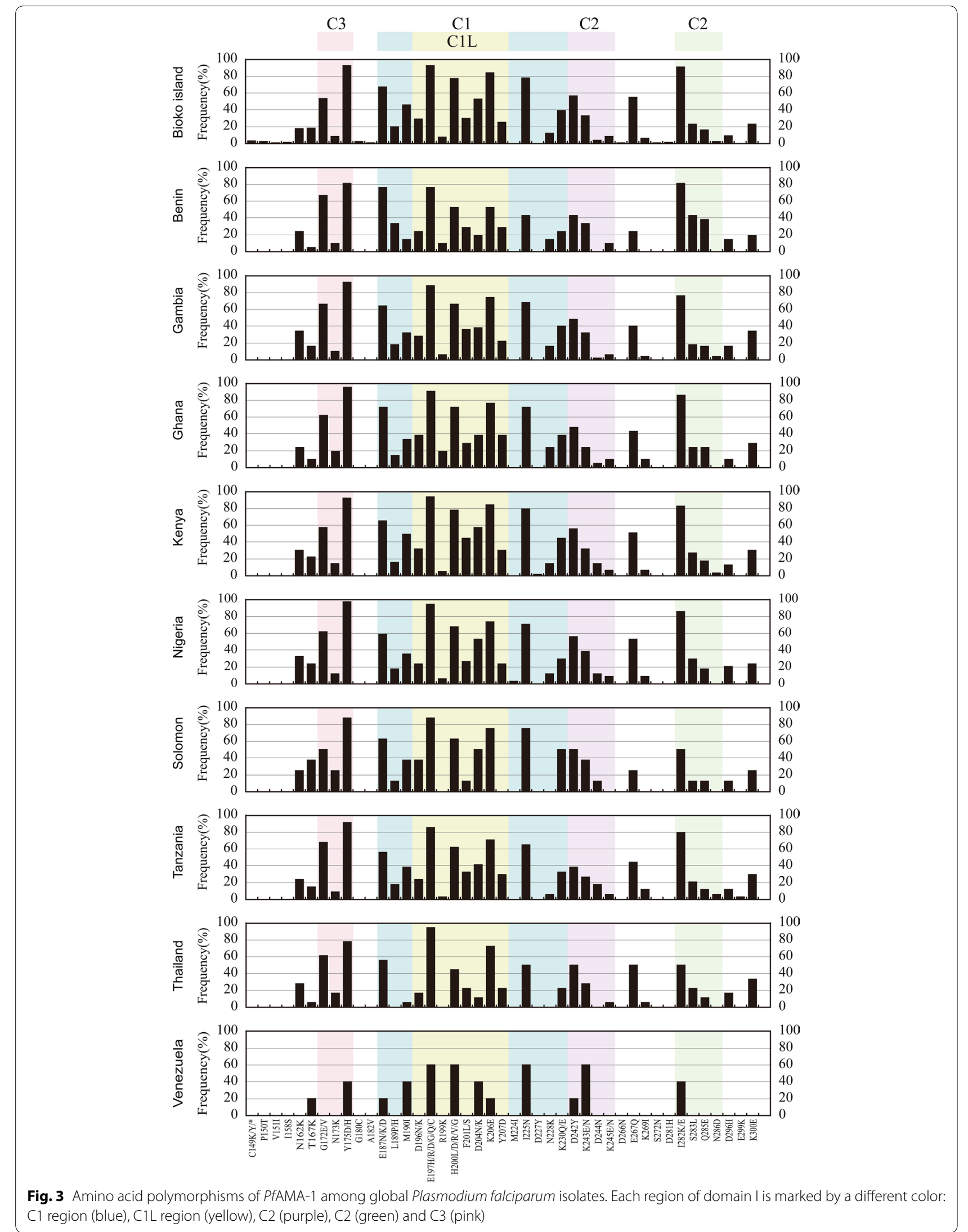


Table 1 DNA sequence polymorphism and tests of neutrality at PfAMA-1 among Plasmodium falciparum Bioko Island isolates

\begin{tabular}{|c|c|c|c|c|c|c|c|c|c|}
\hline Fragment & $\mathrm{Nt} / \mathrm{bp}$ & $S$ & $\begin{array}{l}\text { Total no. } \\
\text { of mutations }\end{array}$ & $\mathrm{K}$ & $\mathrm{H}$ & $\mathrm{Hd} \pm \mathrm{SD}$ & $\pi \pm S D$ & $\mathrm{dN}-\mathrm{dS}$ & Tajima's D \\
\hline Domain I & $445-906$ & 56 & 64 & 12.795 & 131 & $0.9911 \pm 0.0017$ & $0.02776 \pm 0.00042$ & 0.0327 & $0.56734(P>0.10)$ \\
\hline C1 & $559-693$ & 23 & 29 & 7.489 & 87 & $0.976 \pm 0.003$ & $0.05548 \pm 0.00084$ & 0.0641 & $1.49861(P>0.10)$ \\
\hline $\mathrm{C} 1 \mathrm{~L}$ & $586-621$ & 15 & 18 & 4.469 & 52 & $0.937 \pm 0.006$ & $0.12413 \pm 0.00214$ & 0.1378 & $1.24277(P>0.10)$ \\
\hline \multirow[t]{2}{*}{$\mathrm{C} 2$} & $724-735$ & 6 & 6 & 1.421 & 11 & $0.777 \pm 0.018$ & $0.11840 \pm 0.00490$ & 0.1669 & $0.82057(P>0.10)$ \\
\hline & $844-858$ & 5 & 5 & 0.957 & 6 & $0.599 \pm 0.031$ & $0.06381 \pm 0.00416$ & 0.0834 & $0.26245(P>0.10)$ \\
\hline C3 & $514-525$ & 3 & 5 & 0.925 & 7 & $0.692 \pm 0.020$ & $0.07711 \pm 0.00361$ & 0.1018 & $0.19039(P>0.10)$ \\
\hline
\end{tabular}

$S$ segregating sites, $K$ average number of pairwise nucleotide differences, $H$ number of haplotypes, $H d$ haplotype diversity, $\pi$ observed average pairwise nucleotide diversity, $d N$ rate of non-synonymous mutations, $d S$ rate of synonymous mutations

${ }^{*} \mathrm{P}<0.05,{ }^{* *} \mathrm{P}<0.02$

Table 2 Estimates of DNA sequence polymorphism and tests of neutrality at PfAMA-1 among other global plasmodium falciparum isolates

\begin{tabular}{|c|c|c|c|c|c|c|c|c|c|}
\hline Isolate & $\mathrm{S}$ & $\begin{array}{l}\text { Total no. } \\
\text { of mutations }\end{array}$ & $\mathrm{K}$ & $\mathrm{H}$ & $\mathrm{Hd} \pm \mathrm{SD}$ & $\pi \pm S D$ & Tajima's D & Fu and Li's D & Fu and Li's F \\
\hline $\begin{array}{l}\text { Bioko Island } \\
\quad(n=214)\end{array}$ & 56 & 64 & 12.795 & 131 & $0.9911 \pm 0.0017$ & $0.02776 \pm 0.00042$ & $0.56734(P>0.10)$ & $\begin{array}{r}-0.27228 \\
(P>0.10)\end{array}$ & $0.12925(P>0.10)$ \\
\hline Ghana $(n=37)$ & 37 & 41 & 12.555 & 22 & $0.967 \pm 0.012$ & $0.02717 \pm 0.00090$ & $1.01706(P>0.10)$ & $1.29910(P>0.10)$ & $1.42603(P>0.10)$ \\
\hline Tanzania $(n=62)$ & 40 & 45 & 12.460 & 35 & $0.972 \pm 0.008$ & $0.02697 \pm 0.00061$ & $1.01442(P>0.10)$ & $1.59904(P<0.05)$ & $\begin{array}{l}1.64786 \\
\quad(0.10>P>0.05)\end{array}$ \\
\hline Nigeria $(n=51)$ & 38 & 42 & 12.409 & 35 & $0.979 \pm 0.008$ & $0.02686 \pm 0.00068$ & $1.13803(P>0.10)$ & $1.53256(P<0.05)$ & $\begin{array}{l}1.65608 \\
\quad(0.10>P>0.05)\end{array}$ \\
\hline Solomon $(n=50)$ & 31 & 32 & 11.791 & 9 & $0.844 \pm 0.021$ & $0.02552 \pm 0.00074$ & $2.19486(P<0.05)$ & $\begin{array}{l}1.34414 \\
\quad(0.10>P>0.05)\end{array}$ & $1.96178(P<0.02)$ \\
\hline Gambia $(n=114)$ & 42 & 48 & 12.267 & 51 & $0.970 \pm 0.006$ & $0.02655 \pm 0.00051$ & $1.12103(P>0.10)$ & $1.22880(P>0.10)$ & $1.42163(P>0.10)$ \\
\hline Kenya $(n=129)$ & 42 & 48 & 12.886 & 64 & $0.980 \pm 0.004$ & $0.02789 \pm 0.00038$ & $1.42081(P>0.10)$ & $1.71986(P<0.02)$ & $1.91516(P<0.05)$ \\
\hline Benin $(n=23)$ & 34 & 37 & 11.605 & 22 & $0.993 \pm 0.014$ & $0.02577 \pm 0.00237$ & $0.65437(P>0.10)$ & $1.22364(P>0.10)$ & $1.22668(P>0.10)$ \\
\hline $\begin{array}{l}\text { Venezuela } \\
\qquad(n=30)\end{array}$ & 15 & 15 & 5.617 & 6 & $0.482 \pm 0.101$ & $0.01216 \pm 0.00228$ & $1.65975(P>0.10)$ & $1.14686(P>0.10)$ & $\begin{array}{l}1.53677 \\
\quad(0.10>P>0.05)\end{array}$ \\
\hline Thailand $(n=80)$ & 32 & 34 & 11.428 & 19 & $0.919 \pm 0.012$ & $0.02474 \pm 0.00067$ & $2.11203(P<0.05)$ & $0.83255(P>0.10)$ & $\begin{array}{l}1.58398 \\
\quad(0.10>P>0.05)\end{array}$ \\
\hline
\end{tabular}

$S$ segregating sites, $K$ average number of pairwise nucleotide differences, $H$ number of haplotypes, $H d$ haplotype diversity, $\pi$ observed average pairwise nucleotide diversity

a similar pattern for Tajima's D across the board, albeit some differences were identified among DIs with different geographical origins (Fig. 4b).

\section{Recombination and linkage disequilibrium}

The minimum number of recombination events between adjacent polymorphic sites $(\mathrm{Rm})$ for DIs of Bioko PfAMA-1 was estimated to be 21 . The R values between adjacent sites $(\mathrm{Ra})$ and per gene $(\mathrm{Rb})$ were 0.2646 and 122, respectively. Possible recombination events were also identified in DIs of other global PfAMA-1 genes. The highest $\mathrm{R}$ values were predicted for DIs of African PfAMA-1 (Nigeria and Kenya) populations, whereas the lowest $\mathrm{R}$ values were predicted for DIs of Venezuela
PfAMA-1 isolates (Table 3). The LD index $\left(\mathrm{R}^{2}\right)$ for DIs of global PfAMA-1 genes also decreased with increasing distance across this gene (Additional file 2).

\section{Nucleotide differentiation among Dls of other global PfAMA-1}

To investigate the degree of gene flow and genetic differentiation among DIs of global PfAMA-1 sequences, Fst values were evaluated for DIs from different geographical P. falciparum populations deposited in GenBank (Table 4). Fst values between different geographical PfAMA-1 populations varied from $0.00317(-, \mathrm{P}>0.05)$ between Kenya and Nigeria to $0.32747(+, \mathrm{P}<0.05)$ between Venezuela and Benin, except negative values. 

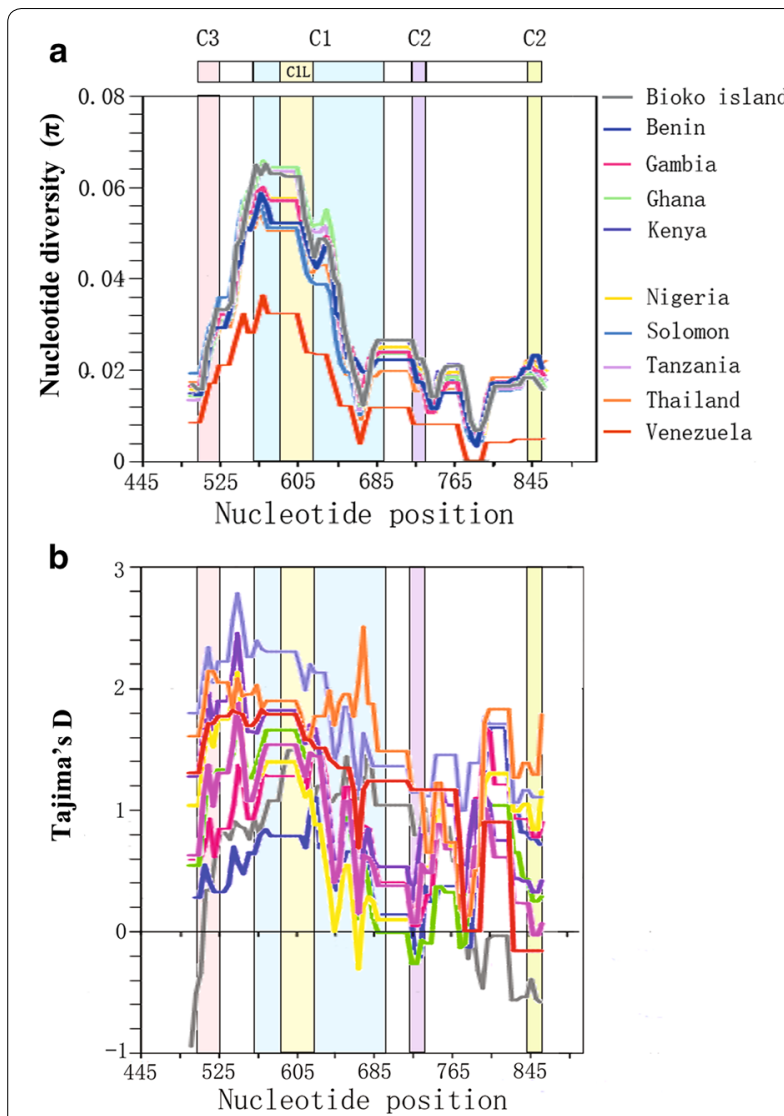

Fig. 4 Nucleotide diversity and natural selection of Dls of global PfAMA-1 sequences. a Nucleotide diversity. Sliding window plot analysis shows the nucleotide diversity $(\pi)$ value across Dls of other global PfAMA-1 sequences. A window size of 100 bp and a step size of 5 bp were used. $\mathbf{b}$ Natural selection. Sliding window calculation of Tajima's D statistic was performed for global PfAMA-1 genes. A window size of 100 and a step size of 5 were used. Bioko Island, jasper; Benin, light blue; Gambia, pink; Ghana, green; Kenya, deep blue; Nigeria, yellow; Solomon, purple; Tanzania, gray; Thailand, orange; Venezuela, red

Three negative values appeared in the Fst analysis, which may be due to the close geographical location of the sample source and the short sequence interval analysed in this study.

\section{Haplotype network analysis}

Haplotype network analysis of PfAMA-1 haplotypes from global $P$. falciparum populations showed a dense network with pretty complex relationships (Fig. 5). A total of 268 haplotypes were identified in 790 PfAMA-1 sequences, of which $66.04 \%$ was singleton. Haplotype prevalence ranged from 0.12 to $4.17 \%$. The most prevalent haplotype was haplotype 48 (Hap_48) with a frequency of 4.17\%. Haplotypes 4, 5, 34, 58, 72, 132 and 141 (Hap_4, $5,34,58,72,132$ and 141) were other major haplotypes
Table 3 Comparison of recombination events of domain I between other global Pf AMA-1 genes

\begin{tabular}{llll}
\hline & Ra & Rb & Rm \\
\hline Bioko Island & 0.2646 & 122 & 21 \\
Ghana & 0.1813 & 83.6 & 12 \\
Tanzania & 0.2191 & 101 & 12 \\
Nigeria & 0.2321 & 107 & 13 \\
Solomon & 0.0397 & 18.3 & 6 \\
Gambia & 0.1666 & 76.8 & 15 \\
Kenya & 0.2321 & 107 & 16 \\
Benin & 0.0865 & 39.9 & 10 \\
Venezuela & 0.000 & 0.001 & 1 \\
Thailand & 0.0751 & 34.6 & 8 \\
\hline
\end{tabular}

The $\mathrm{R}$ and $\mathrm{Rm}$ were estimated excluding the sites containing alignment gaps or those segregating for three nucleotides. The $R$ was computed using $R=4 \mathrm{Nr}$, where $\mathrm{N}$ is the population size and $\mathrm{r}$ is the recombination rate per sequence (per gene) $n$, number of isolates; Ra, recombination parameter between adjacent sites; $\mathrm{Rb}$, recombination parameter for entire gene; $\mathrm{Rm}$, minimum number of recombination events between adjacent sites

with high prevalence (2.53 to $3.29 \%$ ). Only Haplotype_48 (Hap_48) contained haplotypes from three continents. Haplotypes_58, 140, 141, 142 and 145 (Hap_58, 140, 141, 142 and 145) were composed of haplotypes from two continents (Asian and African populations). Haplotypes from Venezuela were more likely to be distributed alone (Hap_132, 133, 134 and 135). Haplotypes from Bioko Island were mostly scattered with no particular distribution pattern (red pie, Fig. 5).

\section{Association between natural selection and host immune pressure}

The selective pressure of host immunity on DIs was evaluated by analyzing genetic polymorphisms in predicted RBC-binding sites, B-cell epitopes and IUR regions. Results showed that most amino acid changes caused by SNPs were found in predicted RBC-binding sites, B-cell epitopes or IUR regions of PfAMA-1 (Fig. 6a). Seven amino acid changes were found in predicted RBC-2 binding sites, two of which (Y175D/H (92.37\%), E187 N/K/D $(67.18 \%))$ were high frequency and two (G180C, A182 V) were novel sites. Five and sixteen amino acid changes were found in predicted $\mathrm{B}$ cell- 3 and 4 regions, respectively. Most of them were commonly identified in global PfAMA-1 isolates, each of these two predicted regions has a new amino acid change, I158S was in the predicted B cell- 3 region and D266 N was in the predicted B cell-4 region. Also, four amino acid changes were found in the predicted IUR-2 (I225 N, N228 K, K230Q/E and D242Y) and IUR-3 (D266 N, E267Q, K269I and S272 N) regions, respectively, and were all commonly identified in global PfAMA-1. The $\pi$ value and Tajima's D value 
Table 4 Pairwise Fst estimates for DI of PfAMA-1

\begin{tabular}{|c|c|c|c|c|c|c|c|c|c|c|}
\hline & Bioko Island & Benin & Gambia & Tanzania & Ghana & Nigeria & Kenya & Thailand & Venezuela & Solomon \\
\hline Bioko Island & & + & + & + & - & - & + & + & + & + \\
\hline Benin & 0.04597 & & - & + & - & + & + & + & + & + \\
\hline Gambia & 0.01824 & 0.01760 & & - & - & - & - & + & + & + \\
\hline Tanzania & 0.01218 & 0.03052 & 0.00330 & & - & - & - & + & + & + \\
\hline Ghana & 0.00548 & 0.01743 & 0.00765 & -0.00464 & & - & - & - & + & + \\
\hline Nigeria & 0.00812 & 0.05139 & 0.00582 & 0.00439 & 0.01375 & & - & + & + & + \\
\hline Kenya & 0.00850 & 0.03536 & 0.00809 & -0.00623 & -0.00267 & 0.00317 & & + & + & + \\
\hline Thailand & 0.03965 & 0.03866 & 0.03152 & 0.03677 & 0.01610 & 0.03908 & 0.03506 & & + & + \\
\hline Venezuela & 0.18281 & 0.32747 & 0.20991 & 0.23285 & 0.25141 & 0.20272 & 0.20219 & 0.23502 & & + \\
\hline Solomon & 0.05101 & 0.07254 & 0.03205 & 0.04926 & 0.05055 & 0.04079 & 0.04068 & 0.04134 & 0.18970 & \\
\hline
\end{tabular}

Fst values are shown in the lower left quadrant and $\mathrm{P}$ values $(+: \mathrm{P}<0.05)$ are shown in the upper right quadrant. Fst, a measure of genetic differentiation between populations (range from 0 to +1 ).

of these regions were calculated in the different countries (Fig. 6b). In particular, high levels of $\pi$ were predicted for RBC-2 region, and the value varied little by different country except Venezuela (0.0333). Tajima's $\mathrm{D}$ values for the predicted RBC-2, B cell-3, 4 and IUR-3 regions were all positive, indicating balancing selection. Meanwhile, Tajima's D values for predicted IUR-2 were negative (Benin and Nigeria) (Fig. 6b). The several predicted regions contained major polymorphic amino acid changes in global PfAMA-1 isolates, and five of eight novel sites were found in these regions (Fig. 6a).

\section{Discussion}

The World Malaria Report 2018 draws on data from 87 countries and areas with ongoing malaria transmission. These reports show that after an unprecedented period of success in global malaria control, progress has stalled [2]. This study provides useful information for the prevention and control of P. falciparum on Bioko Island by analyzing the DI region of AMA-1 gene for a P. falciparum vaccine, as well as characterize the genetic polymorphism and molecular evolution. Although the frequencies of these new mutation sites are not very high, four of them are distributed in B cell-3 (I158T), RBC-2 (G180C, A182V) and B cell-4 (D266N), respectively. The predicted results showed that all 8 mutations are likely to affect the structure and function of PfAMA-1 (probably damaging). Most of these proteins are distributed in the corner area. Whether these mutations cause changes in protein structure and function and affect the binding with human host protein remains to be verified (Table 5, Fig. 2). Moreover, it was found that mutations in DI domain, especially in the $\mathrm{C} 1, \mathrm{C} 1 \mathrm{~L}, \mathrm{C} 2$ and $\mathrm{C} 3$ regions, are highly correlated with the pathogenicity of the host after parasite antigen escape and infection [49]. Nucleotide sequence analysis of these 214 PfAMA-1 sequences from Bioko populations compared to PfAMA-1 from P. falciparum clone 3D7 (GenBank Accession Number U65407) revealed 131 different haplotypes. Most amino acid changes identified from Bioko PfAMA-1 isolates were clustered in $\mathrm{C} 1$ region, especially the $\mathrm{C} 1 \mathrm{~L}$ region, which is consistent with previous reports on the DI region mutations of PfAMA-1 from other endemic areas $[45,50]$. Overall distribution patterns and frequencies of amino acid changes found on Bioko Island were similar to those of other PfAMA-1 changes found globally, but several differences between Bioko Island and other global PfAMA-1 isolates have also been identified in this study. The implication of these geographic differences is not entirely clear. Considering that only the DI region and a limited number of PfAMA-1 isolates in each geographical area were analysed in this study, these amino acid changes and their different frequencies may not be statistically significant. In fact, many of these amino acid changes in other PfAMA-1 genes were generally found and distributed globally in other PfAMA-1 sequences, although their frequencies varied between and among populations. Therefore, to better understand the polymorphism of nucleotide and amino acid of PfAMA-1 on Bioko Island, more PfAMA-1 sequences are required.

The $\pi$ value for global PfAMA-1 varies, ranging from 0.01216 (Venezuela) to 0.02789 (Kenya). The $\pi$ values of African PfAMA-1 sequences (Bioko Island, Ghana, Tanzania, Nigeria, Gambia, Kenya and Benin) are higher than those in South American and Asian PfAMA-1 sequences. The $\pi$ values are higher on Bioko Island (0.02776) than in other African countries except Kenya (0.02789). Indeed, nucleotide diversity was not distributed throughout Bioko Island PfAMA-1. The C2 (844-858 bp) and C3 (514-525 bp) regions show low levels of nucleotide diversity, indicating that these regions might be more conserved, with a lower frequency of polymorphisms. 


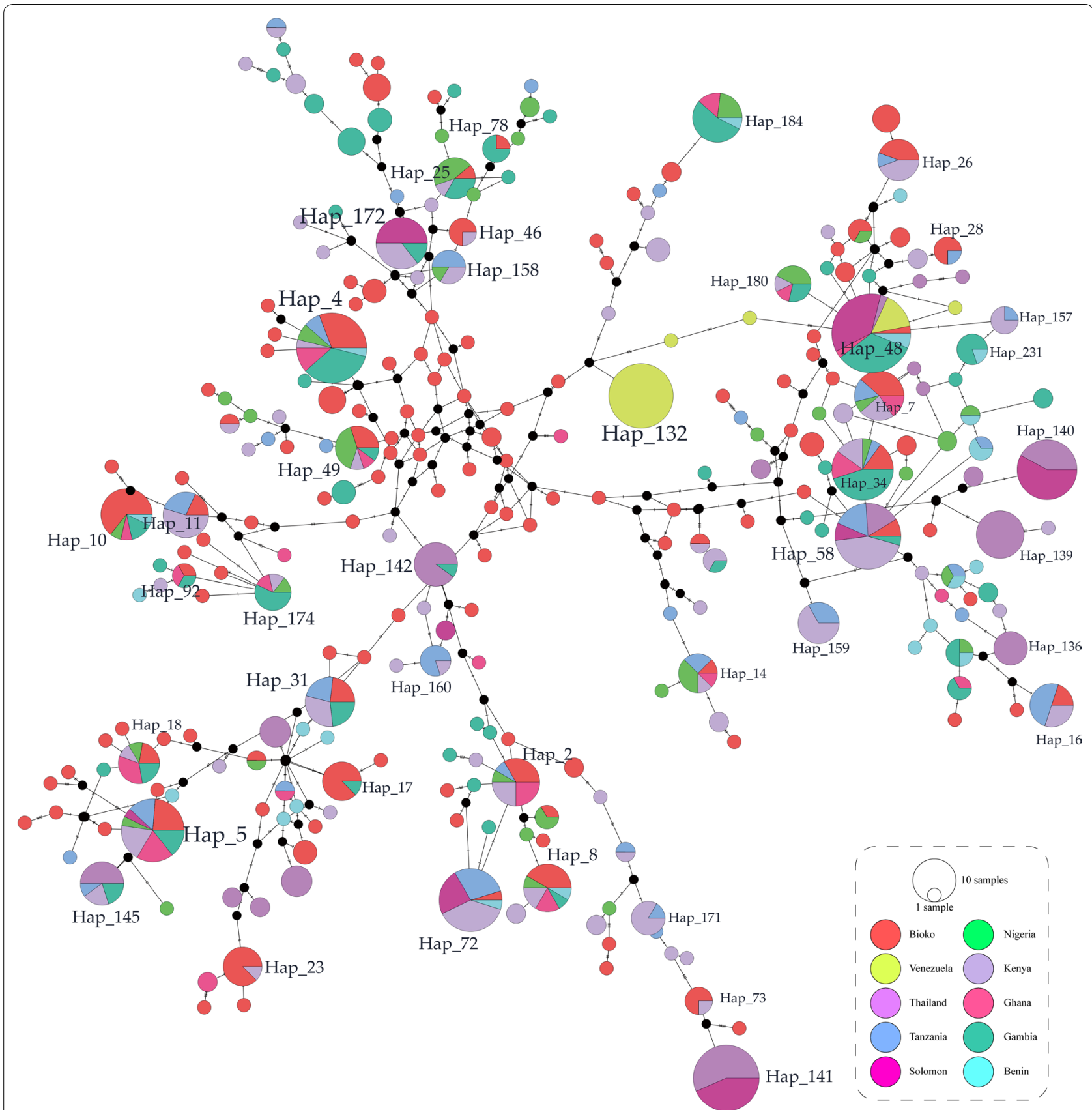

Fig. 5 Network analysis of global PfAMA-1 haplotypes. Bioko Island, yellow; Benin, light blue; Gambia, orange; Ghana, green; Kenya, light pink; Nigeria, deep blue; Solomon, red; Tanzania, deep pink; Thailand, brown; Venezuela, jasper

Moreover, much higher values of nucleotide diversity are observed in the C1L (586-621 bp) and another C2 region (724-735 bp) regions, indicating that a higher frequency of polymorphisms occurs at these regions. Indeed, DI, especially the $\mathrm{C} 1$ and $\mathrm{C} 1 \mathrm{~L}$ regions, are targets of the host's immune system. The high number of DI polymorphisms in the Bioko Island population suggests that this region is under the selection of host immune pressure during evolution. Previous studies on disease-blocking vaccines using PfAMA-1 monoclonal antibody showed that amino acid mutations in the $C 1$ region (197/200/201/204/225) of PfAMA-1 could block the binding of monoclonal antibody to PfAMA-1, thereby inhibiting the effectiveness of the vaccine. In this study, the $\mathrm{C} 1$ region showed a high number of polymorphisms at the gene and amino acid levels, suggesting that the above region may affect the 


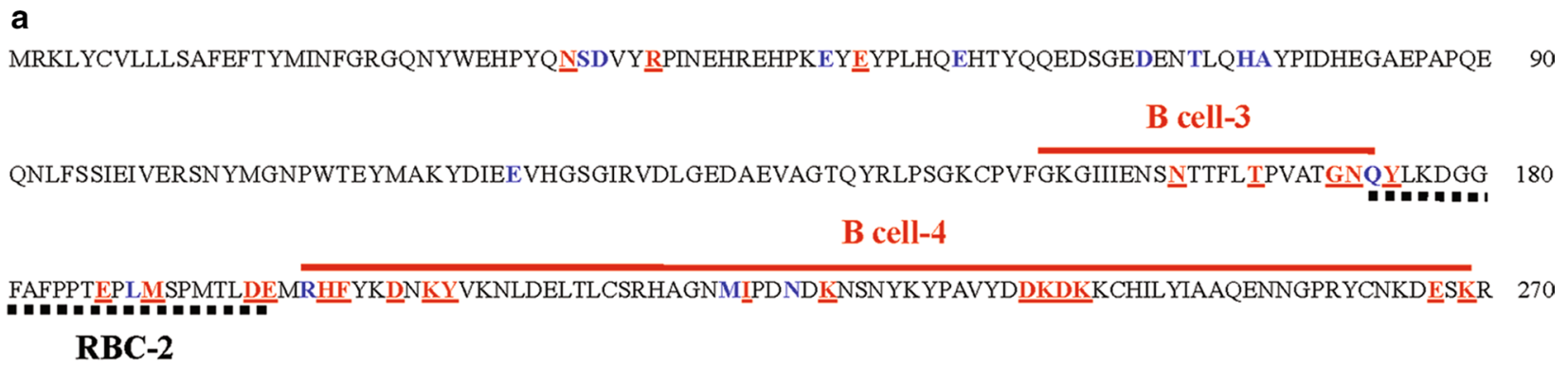

b

\begin{tabular}{|c|c|c|c|c|c|c|c|c|c|c|c|c|c|c|c|c|c|c|c|c|}
\hline \multirow{3}{*}{\begin{tabular}{|l|} 
\\
$\begin{array}{l}\text { RBC-binding } \\
\text { region }\end{array}$ \\
\end{tabular}} & \multicolumn{2}{|c|}{ Bioko } & \multicolumn{2}{|c|}{ Benin } & \multicolumn{2}{|c|}{ Gambia } & \multicolumn{2}{|c|}{ Ghana } & \multicolumn{2}{|c|}{ Kenya } & \multicolumn{2}{|c|}{ Migeria } & \multicolumn{2}{|c|}{ Solom on } & \multicolumn{2}{|c|}{ Tanzania } & \multicolumn{2}{|c|}{ Thail and } & \multicolumn{2}{|c|}{ Venezuela } \\
\hline & $\pi$ & TD & $\pi$ & $\mathrm{rD}$ & $\pi$ & $\mathrm{TD}$ & $\pi$ & TD & $\pi$ & TD & $\pi$ & $\mathrm{TD}$ & $\pi$ & $\mathrm{Tn}$ & $\pi$ & $\mathrm{TD}$ & $\pi$ & In & $\pi$ & TD \\
\hline & & \multirow[b]{2}{*}{0.7767} & \multirow[b]{2}{*}{0.050} & \multirow[b]{2}{*}{0.9611} & \multirow[b]{2}{*}{0.0491} & \multirow[b]{2}{*}{1.8069} & \multirow[b]{2}{*}{0.0506} & \multirow[b]{2}{*}{ 2. 0692} & \multirow[b]{2}{*}{0.0521} & \multirow[b]{2}{*}{2.5677} & \multirow[b]{2}{*}{$0.0494 \mid$} & \multirow[b]{2}{*}{2.1866} & \multirow[b]{2}{*}{0.0534} & \multirow[b]{2}{*}{2.5757} & \multirow[b]{2}{*}{0.0521} & \multirow[b]{2}{*}{2.1125} & & & \multirow[b]{2}{*}{0.0333} & \multirow[b]{2}{*}{1.6928} \\
\hline RBC-2 & 0.0517 & & & & & & & & & & & & & & & & 0.0443 & 1.9546 & & \\
\hline \multicolumn{21}{|l|}{\begin{tabular}{|l|} 
B-cell \\
epitopes
\end{tabular}} \\
\hline B-cell 3 & 0.0202 & 0.6095 & 0.0177 & 0.0126 & 0.0207 & 0.4669 & 0.0196 & 0.8658 & 0.0199 & 1.4924 & 0.0213 & 1.3268 & 0.0235 & 1.687 & 0.0186 & 0.9652 & 0.0228 & 1.7846 & $0.0065 \mid$ & 1.0812 \\
\hline B-cell 4 & 0.0288 & 1.1132 & 0.0235 & 0.3868 & 0.026 & 0.659 & 0.0283 & 0.5157 & 0.0286 & 0.7936 & 0.0259 & 0.2644 & 0.021 & 1.6385 & 0.0269 & 0.5486 & 0.0216 & 1.4891 & $0.0109 \mid$ & 1.5631 \\
\hline \multicolumn{21}{|l|}{ IUR } \\
\hline IUR-2 & 0.0244 & 0.9111 & 0.0232 & -0.006 & 0.0259 & 0.3766 & 0.0241 & 0.0813 & 0.0231 & 0.1466 & 0.0299 & -0.309 & 0.0202 & 1.1271 & 0.0222 & 0.1527 & 0.018 & 2.0568 & $0.0071 \mid$ & 0.6838 \\
\hline IUR-3 & 0.0175 & 0.0249 & 0.0093 & 0.4803 & 0.0168 & 1.1051 & 0.0183 & 0.9518 & 0.0164 & 1.0835 & $0.0157 \mid$ & 0.6839 & 0.0116 & 1.3896 & 0.0154 & 0.7 & 0.0136 & 0.5071 & 0 & 1 \\
\hline
\end{tabular}

Fig. 6 Association between natural selection and host immune pressure. a Positions of amino acid changes found in global PfAMA-1 and predicted RBC-binding sites, B-cell epitopes and IUR regions. Predicted RBC-binding sites, B-cell epitopes and IUR regions are presented by dotted black lines, red lines and bold blue lines, respectively. Polymorphic amino acid residues commonly identified in global PfAMA-1 are marked as bold red with underline. The less commonly identified amino acid changes are shown as bold blue. $\mathbf{b}$ Nucleotide diversity and natural selection analysis. Nucleotide diversity $(\pi)$ and Tajima's D (TD) values for each RBC-binding sites, B-cell epitopes and IUR regions in DI were analysed using Dnasp 6.0 program

effectiveness of vaccines. Although the values are different among and between other global PfAMA-1 isolates, highly similar distribution patterns of nucleotide diversity have been detected in global PfAMA-1 genes analysed in this study, strongly indicating that these PfAMA-1 genes might share highly similar nucleotide diversity. The $\mathrm{dN}-\mathrm{dS}$ value for Bioko Island PfAMA-1 DIs is positive, suggesting involvement of balancing selection. Also, the $\mathrm{dN}-\mathrm{dS}$ value for all regions of DI is positive, indicating positive natural selection throughout DIs. The positive
Tajima's D values for Bioko Island PfAMA-1 also suggested that this gene might have evolved under balancing selection. In the sliding plot analysis of Tajima's D, general patterns of Tajima's D values across Bioko Island PfAMA-1 were similar to those of other PfAMA-1 genes, although differences among and between PfAMA-1s from different countries were also found. Regardless of the slight difference in values, $\mathrm{C} 1, \mathrm{C} 2$ and $\mathrm{C} 3$ regions all shared pretty similar distribution patterns of Tajima's D values, strongly indicating that these regions might be 
Table 5 Function prediction scores of eight novel sites

\begin{tabular}{llllll}
\hline Novel sites & Frequency (\%) & Score (HumDiv) & Sensitivity & Specificity $^{\mathbf{a}}$ & Classification $^{\mathbf{b}}$ \\
\hline P150T & 2.29 & 1.000 & 0.00 & 1.00 & Probably damaging \\
V151I & 0.76 & 0.714 & 0.86 & 0.92 & Probably damaging \\
I158S & 1.53 & 0.998 & 0.27 & 0.99 & Probably damaging \\
G180C & 2.29 & 1.000 & 0.00 & 1.00 & Probably damaging \\
A182V & 0.76 & 1.000 & 0.00 & 1.00 & Probably damaging \\
D266N & 3.82 & 0.991 & 0.71 & 0.97 & Probably damaging \\
S272N & 0.76 & 0.846 & 0.83 & 0.93 & Probably damaging \\
D281H & 1.53 & 1.000 & 0.00 & 1.00 & Probably damaging
\end{tabular}

a HumDiv is preferred model for evaluating rare alleles, dense mapping of regions identified by genome-wide association studies, and analysis of natural selection

b Qualitative ternary classfication appraised at 5\%/10\% (HumDiv) FPR thresholds ("benign", "possibly damaging”, "probably damaging”)

major targets for the host immune response. Fu and Li's $\mathrm{D}$ and $\mathrm{F}$ tests also provide enough evidence for balancing selection of PfAMA-1 on Bioko Island. These values suggest that PfAMA-1 DIs from Bioko Island populations are highly polymorphic with a strong selection force acting on it, similar to other global PfAMA-1 genes. In addition to natural selection, recombination also contributes to the diversity of PfAMA-1. Meiotic recombination that occurs between the adjacent polymorphic sites is responsible for the high allelic diversity in DI [45]. These results also indicate that a high level of recombination events have occurred in PfAMA-1 isolates on Bioko Island. High levels of recombination in PfAMA-1 from different geographical isolates have been previously reported $[28,35$, $45,46,50-54]$. High recombination events were found in Bioko Island PfAMA-1 sequences compare to samples from other geographical areas. This might be due to special island location of Bioko region, which attribute an opportunity to PfAMA-1 to undergo more recombination. Moreover, the incidence of recombination events in African countries is also generally higher than other areas, confirming the previous conclusions of recombination events. This is supported by the decline of the LD index $\mathrm{R}^{2}$ with increasing nucleotide distance in PfAMA-1 isolates on Bioko Island, consistent with previous reports $[28,45,46,50-54]$. In conclusion, recombination is the most important factor generating genetic diversity in global PfAMA-1 populations.

The Fst value is one of the most useful methods for analyzing overall genetic differentiation (range from 0 to $+1)$. Fst values at each locus are considered as having no differentiation (0), low genetic differentiation (0-0.05), moderate differentiation $(0.05-0.15)$ or high differentiation (0.15-0.25) [54]. The Fst values for PfAMA-1 DIs on Bioko Island show a lower level of genetic differentiation than that of PfAMA-1 in other geographical populations except Benin, which occurs at a moderate level, as well as the values of Fst for most Asian and African countries. Also, a high level of genetic differentiation is found between Bioko Island and Venezuela. All of these indicate that Fst values between PfAMA-1 populations belonging to the same geographical area are relatively low. The negative values in the table were considered because DI of PfAMA-1 is too limited. However, Bioko Island shows a high Fst value for Venezuela (0.22087), providing strong evidence for geographical isolation and population segmentation in these regions. Total Fst values of DI between and among global PfAMA-1 isolates are in the low to medium differentiation range, indicating that PfAMA-1 has limited genetic differentiation among other parasite populations around the world.

It is important to understand the haplotype network of PfAMA-1 on Bioko Island with other global areas in order to develop a globally effective malaria vaccine based on this gene. A total of 296 different haplotypes were identified by network analysis of 790 PfAMA-1 sequences. Haplotype network analysis shows that haplotypes on Bioko Island are scattered among other haplotypes from different countries, which is consistent with a previous report [53]. Compared with the African continent, Bioko Island is an independent Island with a special geographical location. It is an offshore Island, with special species diversity and evolution, but very close to seriously affected areas of malaria in West Africa. As a result, the distribution of $P$. falciparum on Bioko Island may to have no differences with the continent of Africa, but the distribution of the different geographic strains on the island is unclear. Therefore, the genetic diversity of PfAMA-1 DI regions on Bioko Island were analysed to carry out a detailed investigation on the distribution of different geographical strains to help understand the situation in this region and provide a reference for drug resistance investigations and medication guidance. Many single haplotypes appear in both clusters, but no pie completely covering all haplotypes was found in all geographic areas in this study. A recent report suggested that mutations in the PfAMA-1 sequence are not necessarily strong predictors of antigenic differences or cross-inhibitory antibody 
activity levels, since not all polymorphic residues contribute equally to antibody generation and escape [47]. Due to the limited diversity of PfAMA-1 antigens, vaccines targeting a small number of PfAMA-1 alleles might be sufficient to cover naturally circulating populations of $P$. falciparum in different endemic areas [33]. These results also indicate that the global genetic diversity of PfAMA-1 is relatively limited, even though substantial geographic differentiation can also be identified among populations. Nevertheless, global PfAMA-1 is undergoing natural selection and high levels of meiotic recombination, which can produce new alleles in a gene population. Therefore, consideration should be given to the development of PfAMA-1-based malaria vaccines using a polyallelic approach to maximize vaccine effectiveness.

To assess the association between host immune pressure and natural selection of PfAMA-1, genetic polymorphisms in predicted B-cell epitopes and IUR regions were analysed. Based on a recent report, detailed information on potential RBC-binding regions, B-cell epitopes and IURs across the ectodomain of PfAMA-1 were obtained [45]. Most amino acid changes found in DI of PfAMA-1 on Bioko Island are predicted to be localized at predicted B-cell epitopes or IUR regions in DI. In this study, B-cell epitopes 3 and 4 show high levels of nucleotide diversity in PfAMA-1 on Bioko Island. Tajima's D values for these predicted B-cell epitopes also suggest that these epitopes are under natural selection. The $\pi$ values of RBC-2 regions in different geographical areas are close to each other, which suggests that the nucleotide diversity of DIs is similar in these regions. In Bioko Island PfAMA-1 isolates analysed in this study, the DI region shows clustering of amino acid polymorphisms in the C1L region. The $\mathrm{C} 1 \mathrm{~L}$ region is located near the hydrophobic pocket of DIs, which affects the binding of inhibitory monoclonal antibodies and thus leads to escape from antibody targeting $[55,56]$. This shows that the important role of natural selection in generating PfAMA-1 gene diversity is very obvious just as the effect of natural selection of $P$. falciparum on Bioko Island, and also supports the idea that natural selection can promote host immune escape in this region $[57,58]$. Therefore, it may be necessary to consider the polymorphism in DI in order to obtain more efficient vaccine components.

\section{Conclusions}

A major problem in the development of effective malaria vaccines is the genetic polymorphism observed in isolates worldwide. PfAMA-1 is one of the most promising malaria vaccine candidates for the blood stage of $P$. falciparum. The overall pattern of nucleotide diversity and distribution of amino acid changes of PfAMA-1 on Bioko Island is similar to those from other global isolates, although several novel amino acid changes are found on Bioko Island. Natural selection to balance across PfAMA-1 and high levels of recombination events observed on Bioko Island and other global PfAMA-1 genes suggest that natural selection and intragenic recombination might be the main drivers of genetic diversity in global PfAMA-1. High levels of nucleotide diversity and natural selection suggest that strong natural selection might have an effect on immune stress-related epitopes of PfAMA-1 hosts. The amino acid changes of DIs in PfAMA-1 on Bioko Island are mainly in predicted B cell epitopes. Although a highly complex haplotype diversity has been found in global populations, genetic diversity of PfAMA-1 between and among global parasite populations remains limited. Follow-up studies such as more samples and the expansion of PfAMA-1 may yield more representative mutation trends. The results of this study ensure the continuous detection of PfAMA-1 nucleotide and amino acid changes in global PfAMA-1, so as to provide more useful information for the development of malaria vaccine.

\section{Supplementary information}

Supplementary information accompanies this paper at https://doi. org/10.1186/s12936-019-2948-y.

Additional file 1: Amino acid sequence polymorphisms of domain I in PFAMA-1 from Bioko Island isolates of P. falciparum.

Additional file 2: Recombination events in global PfAMA-1 genes. Linkage disequilibrium (LD) plots showed a non-random association between nucleotide variants in DI of PfAMA-1 at different polymorphic sites. $\mathrm{R}^{2}$ values were plotted against nucleotide distance using a two-tailed Fisher's exact test for statistical significance.

\section{Abbreviations}

PfAMA-1: Plasmodium falciparum apical membrane antigen-1; DI: domain I; CSP: circumsporozoite protein; DBP: Duffy-binding protein; MSP-1: merozoite surface protein-1; TRAP: thrombospondin-related anonymous protein; BIMCP: The Bioko Island Malaria Control Project; $\mathrm{S}$ : numbers of segregating sites; $\mathrm{H}$ : haplotypes; Hd: haplotype diversity; $\pi$ : nucleotide diversity; $\mathrm{K}$ : average number of pair-wise nucleotide differences within a population; $\mathrm{dN}$ : values of nonsynonymous; dS: values of synonymous; $R$ : recombination parameters; Rm: minimum number of recombination events; LD: linkage disequilibrium; Fst: fixation index.

\section{Acknowledgements}

The authors thank the Department of Health of Guangdong Province and the Department of Aid to Foreign Countries of the Ministry of Commerce of the People's Republic of China for their help. Special thanks to Stanley Li Lin for helping to revise and polish the language of the manuscript.

\section{Authors' contributions}

Field work was performed on Bioko Island, EG. Laboratory work was conducted at Shantou University Medical College, XYL, HYH and YNW carried out molecular studies and performed statistical analyses; YNW, ML and HBC conceived and supervised molecular analyses and participated in the paper drafting; JTC, DDX, YLW, CSE, UME, JLW, DYX, ZMC and YLC participated in statistical analyses; YNW, ML and HBC conceived of the study, participated in 
its design and helped draft and critically analysed the manuscript. All authors read and approved the final manuscript.

\section{Funding}

This study was supported by the Natural Science Foundation of Guangdong Province to Jiang-Tao Chen (Grant No. 2016A03031311).

\section{Availability of data and materials}

The datasets supporting the conclusions of this article are included with in the article.

\section{Ethics approval and consent to participate}

Participants in the clinical study provided written informed consent before their enrolment, and the study was approved by the institutional ethics committee of Malabo Regional Hospital, Bioko, Equatorial Guinea. All participants received adequate anti-malarial treatment.

\section{Consent for publication}

All authors have given their consent for publication.

\section{Competing interests}

The authors declare that they have no competing interests.

\section{Author details}

${ }^{1}$ Department of Histology and Embryology, Shantou University Medical College, Shantou, Guangdong, People's Republic of China. ${ }^{2}$ School of Food Engineering and Biotechnology, Hanshan Normal University, Chaozhou, Guangdong, People's Republic of China. ${ }^{3}$ Laboratory Medical Centre, Huizhou Municipal Central Hospital, Huizhou, Guangdong, People's Republic of China. ${ }^{4}$ The Chinese Medical Aid Team to the Republic of Equatorial Guinea, Guangzhou, Guangdong, People's Republic of China. ${ }^{5}$ Department of Medical Laboratory, Malabo Regional Hospital, Malabo, Equatorial Guinea. ${ }^{6} 2014$ Clinical Medicine Programme, Shantou University Medical College, Shantou, Guangdong, People's Republic of China.

Received: 17 April 2019 Accepted: 6 September 2019

Published online: 18 September 2019

\section{References}

1. White NJ, Pukrittayakamee S, Hien TT, Faiz MA, Mokuolu OA, Dondorp AM. Malaria. Lancet. 2014;383:723-35.

2. WHO. World malaria report 2018. Geneva: World Health Organization; 2018. p. 210. ISBN: 978-92-4-156565-3.

3. Li J, Chen J, Xie D, Eyi UM, Matesa RA, Ondo Obono MM, et al. Limited artemisinin resistance-associated polymorphisms in Plasmodium falciparum K13-propeller and PfATPase6 gene isolated from Bioko Island, Equatorial Guinea. Int J Parasitol Drugs Drug Resist. 2016;54-9.

4. Cook J, Hergott D, Phiri W, Rivas MR, Bradley J, Segura L, et al. Trends in parasite prevalence following 13 years of malaria interventions on Bioko Island, Equatorial Guinea: 2004-2016. Malar J. 2018;17:62.

5. Lu F, Culleton R, Zhang M, Ramaprasad A, von Seidlein L, Zhou H, et al. Emergence of indigenous artemisinin-resistant Plasmodium falciparum in Africa. N Engl J Med. 2017;376:991-2

6. Richards JS, Beeson JG. The future for blood-stage vaccines against malaria. Immunol Cell Biol. 2009;87:377-90.

7. Chen JT, Li J, Zha GC, Huang G, Huang ZX, Xie DD, et al. Genetic diversity and allele frequencies of Plasmodium falciparum msp1 and msp2 in parasite isolates from Bioko Island, Equatorial Guinea. Malar J. 2018;12:458.

8. Jiang T, Chen J, Fu H, Wu K, Yao Y, Eyi JUM, et al. High prevalence of PfdhfrPfdhps quadruple mutations associated with sulfadoxine-pyrimethamine resistance in Plasmodium falciparum isolates from Bioko Island, Equatorial Guinea. Malar J. 2019:18:101

9. Peterson MG, Marshall VM, Smythe JA, Crewther PE, Lew A, Silva A, et al. Integral membrane protein located in the apical complex of Plasmodium falciparum. Mol Cell Biol. 1989;9:3151-4.

10. Remarque EJ, Faber BW, Kocken $\mathrm{CH}$, Thomas AW. Apical membrane antigen 1: a malaria vaccine candidate in review. Trends Parasitol. 2008:24:74-84.
11. Healer J, Crawford S, Ralph S, McFadden G, Cowman AF. Independent translocation of two micronemal proteins in developing Plasmodium falciparum merozoites. Infect Immun. 2002;70:5751-8.

12. Silvie O, Franetich JF, Charrin S, Mueller MS, Siau A, Bodescot M, et al. A role for apical membrane antigen 1 during invasion of hepatocytes by Plasmodium falciparum sporozoites. J Biol Chem. 2004;279:9490-6.

13. Bannister LH, Hopkins JM, Dluzewski AR, Margos G, Williams IT, Blackman MJ, et al. Plasmodium falciparum apical membrane antigen 1 (PfAMA-1) is translocated within micronemes along subpellicular microtubules during merozoite development. J Cell Sci. 2003;116:3825-34.

14. Healer J, Murphy V, Hodder AN, Masciantonio R, Gemmill AW, Anders $\mathrm{RF}$, et al. Allelic polymorphisms in apical membrane antigen-1 are responsible for evasion of antibody-mediated inhibition in Plasmodium falciparum. Mol Microbiol. 2004;52:159-68.

15. Hodder AN, Crewther PE, Matthew ML, Reid GE, Moritz RL, Simpson RJ, et al. The disulfide bond structure of Plasmodium apical membrane antigen-1. J Biol Chem. 1996;271:29446-52.

16. Udhayakumar V, Kariuki S, Kolczack M, Girma M, Roberts JM, Oloo AJ, et al. Longitudinal study of natural immune responses to the Plasmodium falciparum apical membrane antigen (AMA-1) in a holoendemic region of malaria in western Kenya: Asembo Bay Cohort Project VIII. Am J Trop Med Hyg. 2001;65:100-7.

17. Cortés A, Mellombo M, Masciantonio R, Murphy VJ, Reeder JC, Anders RF. Allele specificity of naturally acquired antibody responses against Plasmodium falciparum apical membrane antigen 1. Infect Immun. 2005;73:422-30.

18. Mitchell GH, Thomas AW, Margos G, Dluzewski AR, Bannister LH. Apical membrane antigen 1, a major malaria vaccine candidate, mediates the close attachment of invasive merozoites to host red blood cells. Infect Immun. 2004;72:154-8.

19. Yap A, Azevedo MF, Gilson PR, Weiss GE, O'Neill MT, Wilson DW, et al. Conditional expression of apical membrane antigen 1 in Plasmodium falciparum shows it is required for erythrocyte invasion by merozoites. Cell Microbiol. 2014;16:642-56.

20. Liu Q, Singla LD, Zhou H. Vaccines against Toxoplasma gondii: status, challenges and future directions. Hum Vaccin Immunother. 2012;8:1305-8.

21. Alexander DL, Mital J, Ward GE, Bradley P, Boothroyd JC. Identification of the moving junction complex of Toxoplasma gondii: a collaboration between distinct secretory organelles. PLoS Pathog. 2005;1:e17

22. Lamarque M, Besteiro S, Papoin J, Roques M, Vulliez-Le Normand B, Morlon-Guyot J, et al. The RON2-AMA1 interaction is a critical step in moving junction-dependent invasion by apicomplexan parasites. PLoS Pathog. 2011;7:e1001276.

23. Srinivasan $P$, Beatty WL, Diouf $A$, Herrera R, Ambroggio X, Moch JK, et al. Binding of Plasmodium merozoite proteins RON2 and AMA1 triggers commitment to invasion. Proc Natl Acad Sci USA. 2011;108:13275-80.

24. Moncunill G, Mayor A, Jiménez A, Nhabomba A, Casas-Vila N, Puyol L, et al. High antibody responses against Plasmodium falciparum in immigrants after extended periods of interrupted exposure to malaria. PLoS ONE. 2013;8:e73624.

25. Murungi LM, Sonden K, Llewellyn D, Rono J, Guleid F, Williams AR, et al. Targets and mechanisms associated with protection from severe Plasmodium falciparum malaria in Kenyan children. Infect Immun. 2016:84:950-63.

26. Narum DL, Ogun SA, Thomas AW, Holder AA. Immunization with parasitederived apical membrane antigen 1 or passive immunization with a specific monoclonal antibody protects BALB/c mice against lethal Plasmodium yoelii YM blood-stage infection. Infect Immun. 2000;68:2899-906.

27. Cortes A, Mellombo M, Mueller I, Benet A, Reeder JC, Anders RF. Geographical structure of diversity and differences between symptomatic and asymptomatic infections for Plasmodium falciparum vaccine candidate AMA1. Infect Immun. 2003;71:1416-26.

28. Garg S, Alam MT, Das MK, Dev V, Kumar A, Dash AP, et al. Sequence diversity and natural selection at domain I of the apical membrane antigen 1 among Indian Plasmodium falciparum populations. Malar J. 2007;6:154.

29. Polley SD, Chokejindachai W, Conway DJ. Allele frequency-based analyses robustly map sequence sites under balancing selection in a malaria vaccine candidate antigen. Genetics. 2003;165:555-61.

30. Polley SD, Conway DJ. Strong diversifying selection on domains of the Plasmodium falciparum apical membrane antigen 1 gene. Genetics. 2001:158:1505-12. 
31. Polley SD, Mwangi T, Kocken CH, Thomas AW, Dutta S, Lanar DE, et al. Human antibodies to recombinant protein constructs of Plasmodium falciparum apical membrane antigen 1 (AMA1) and their associations with protection from malaria. Vaccine. 2004;23:718-28.

32. Kang JM, Lee J, Cho PY, Moon SU, Ju HL, Ahn SK, et al. Population genetic structure and natural selection of apical membrane antigen-1 in Plasmodium vivax Korean isolates. Malar J. 2015;14:455.

33. Terheggen U, Drew DR, Hodder AN, Cross NJ, Mugyenyi CK, Barry AE, et al. Limited antigenic diversity of Plasmodium falciparum apical membrane antigen 1 supports the development of effective multi-allele vaccines. BMC Med. 2014;12:183.

34. Escalante AA, Grebert HM, Chaiyaroj SC, Magris M, Biswas S, Nahlen BL, et al. Polymorphism in the gene encoding the apical membrane antigen-1 (AMA-1) of Plasmodium falciparum. X. Asembo Bay Cohort Project. Mol Biochem Parasitol. 2001;113:279-87.

35. Moon SU, Na BK, Kang JM, Kim JY, Cho SH, Park YK, et al. Genetic polymorphism and effect of natural selection at domain I of apical membrane antigen-1 (AMA-1) in Plasmodium vivax isolates from Myanmar. Acta Trop. 2010;114:71-5.

36. Volkman SK, Ndiaye D, Diakite M, Koita OA, Nwakanma D, Daniels RF, et al. Application of genomics to field investigations of malaria by the international centers of excellence for malaria research. Acta Trop. 2012;121:324-32

37. Tamura K, Peterson D, Peterson N, Stecher G, Nei M, Kumar S. MEGA5: molecular evolutionary genetics analysis using maximum likelihood, evolutionary distance, and maximum parsimony methods. Mol Biol Evol. 2011:28:2731-9.

38. Rozas J, Ferrer-Mata A, Sánchez-DelBarrio JC, Guirao-Rico S, Librado P, Ramos-Onsins SE, et al. DnaSP 6: DNA sequence polymorphism analysis of large data sets. Mol Biol Evol. 2017;34:3299-302.

39. Nei M, Gojobori T. Simple methods for estimating the number of synonymous and nonsynonymous nucleotide substitutions. Mol Biol Evol. 1986:3:418-26.

40. Tajima F. Statistical method for testing the neutral mutation hypothesis by DNA polymorphism. Genetics. 1989;123:585-95.

41. Fu YX, Li WH. Statistical tests of neutrality of mutations. Genetics. 1993;133:693-709.

42. Adzhubei IA, Schmidt S, Peshkin L, Ramensky VE, Gerasimova A, Bork P, et al. A method and server for predicting missense mutations. Nat Methods. 2010;7:248-9.

43. Excoffier $\mathrm{L}$, Lischer HE. Arlequin suite ver 3.5: a new series of programs to perform population genetics analyses under Linux and Windows. Mol Ecol Res. 2010;10:564-7.

44. Leigh JW, Bryant D. POPART: full-feature software for haplotype Popart construction. Methods Ecol Evol. 2015;6:1110-6.

45. Mehrizi AA, Sepehri M, Karimi F, Djadid ND, Zakeri S. Population genetics, sequence diversity and selection in the gene encoding the Plasmodium falciparum apical membrane antigen 1 in clinical isolates from the southeast of Iran. Infect Genet Evol. 2013;17:51-61.
46. Urquiza M, Suarez JE, Cardenas C, Lopez R, Puentes A, Chavez F, et al. Plasmodium falciparum AMA-1 erythrocyte binding peptides implicate AMA-1 as erythrocyte binding protein. Vaccine. 2000;19:508-13.

47. Kang JM, Lee J, Moe M, Jun H, Lê HG, Kim Tl, et al. Population genetic structure and natural selection of Plasmodium falciparum apical membrane antigen-1 in Myanmar isolates. Malar J. 2018;17:71.

48. Mugyenyi CK, Elliott SR, McCallum FJ, Anders RF, Marsh K, Beeson JG. Antibodies to polymorphic invasion-inhibitory and non-Inhibitory epitopes of Plasmodium falciparum apical membrane antigen 1 in human malaria. PLoS One. 2013;8:e68304.

49. Takala SL, Coulibaly D, Thera MA, Batchelor AH, Cummings MP, Escalante AA, et al. Extreme polymorphism in a vaccine antigen and risk of clinical malaria: implications for vaccine development. Sci Transl Med. 2009;1:2ra5.

50. Zhu X, Zhao Z, Feng Y, Li P, Liu F, Liu J, et al. Genetic diversity of the Plasmodium falciparum apical membrane antigen I gene parasite population from the China-Myanmar border area. Infect Genet Evol. 2016;39:155-62.

51. Basu M, Maji AK, Mitra M, Sengupta S. Natural selection and population genetic structure of domain I of Plasmodium falciparum apical membrane antigen-1 in India. Infect Genet Evol. 2013;18:247-56.

52. Quang ND, Hoa PT, Tuan MS, Viet NX, Jalloh A, Matsuoka H. Polymorphism at the apical membrane antigen 1 gene (AMA1) of the malaria parasite Plasmodium falciparum in a Vietnamese population. Biochem Genet. 2009:47:370-83.

53. Garg S, Alam MT, Das MK, Dev V, Kumar A, Dash AP, et al. Sequence diversity and natural selection at domain I of the apical membrane antigen 1 among Indian Plasmodium falciparum populations. Malar J. 2007;6:154.

54. Eisen DP, Marshall VM, Billman-Jacobe H, Coppel RL. A Plasmodium falciparum apical membrane antigen-1 (AMA-1) gene apparently generated by intragenic recombination. Mol Biochem Parasitol. 1999;100:243-6.

55. Balloux F, Lugon-Moulin N. The estimation of population differentiation with microsatellite markers. Mol Ecol. 2002;11:155-65.

56. Drew DR, Hodder AN, Wilson DW, Foley M, Mueller I, Siba PM, et al. Defining the antigenic diversity of Plasmodium falciparum apical membrane antigen 1 and the requirements for a multi-allele vaccine against malaria. PLoS One. 2012;7:e51023.

57. Coley AM, Parisi K, Masciantonio R, Hoeck J, Casey JL, Murphy VJ, et al. The most polymorphic residue on Plasmodium falciparum apical membrane antigen 1 determines binding of an invasion-inhibitory antibody. Infect Immun. 2006;74:2628-36.

58. Coley AM, Gupta A, Murphy VJ, Bai T, Kim H, Foley M, et al. Structure of the malaria antigen AMA1 in complex with a growth-inhibitory antibody. PLoS Pathog. 2007;3:1308-19.

\section{Publisher's Note}

Springer Nature remains neutral with regard to jurisdictional claims in published maps and institutional affiliations.
Ready to submit your research? Choose BMC and benefit from:

- fast, convenient online submission

- thorough peer review by experienced researchers in your field

- rapid publication on acceptance

- support for research data, including large and complex data types

- gold Open Access which fosters wider collaboration and increased citations

- maximum visibility for your research: over 100M website views per year

At $\mathrm{BMC}$, research is always in progress.

Learn more biomedcentral.com/submissions 